\title{
Simple Model for Wet Granular Materials with Liquid Clusters
}

\author{
NAmiko Mitarai ${ }^{1}$ and Hilzu NaKanishi ${ }^{2}$ \\ 1 Niels Bohr Institute, Blegdamsvej 17, DK-2100, Copenhagen, Denmark. \\ 2 Department of Physics, Kyushu University 33, 812-8581, Fukuoka, Japan.
}

PACS 45.70.-n - Granular systems

PACS 82.70.-y - Disperse systems; complex fluids

PACS $83.80 . \mathrm{Fg}$ - Granular solids

\begin{abstract}
We propose a simple phenomenological model for wet granular media to take into account many particle interaction through liquid in the funicular state as well as two-body cohesive force by a liquid bridge in the pendular state. In the wet granular media with small liquid content, liquid forms a bridge at each contact point, which induces two-body cohesive force due to the surface tension. As the liquid content increases, some liquid bridges merge, and more than two grains interact through a single liquid cluster. In our model, the cohesive force acts between the grains connected by a liquid-gas interface. As the liquid content increases, the number of grains that interact through the liquid increases, but the liquid-gas interface may decrease when liquid clusters are formed. Due to this competition, our model shows that the shear stress has a maximum as a function of the liquid-content.
\end{abstract}

Introduction. - It is well known that just adding small amount of liquid to dry granular materials changes their behaviors drastically [1-3]. Liquid forms bridges be'tween grains, and the bridge induces cohesion between grains due to the surface tension. When the amount of liq, uid is small, liquid bridges are formed between two grains at contact points; such a state is called the pendular state (Fig. 1(a)). As the amount of liquid is increased, several - liquid bridges are connected to form a liquid cluster that includes more than two grains as in Fig. 1(b), which is called the funicular state. As we add the liquid further, ' all the pores will be filled with the liquid. It is called the capillary state if the liquid pressure is still lower than the air pressure, and finally the system becomes the slurry state if the amount of liquid is enough to make the liquid pressure as high as the air pressure.

The liquid content dependence of the mechanical properties of wet granular materials has not been well understood yet. The most studied case is the pendular state, both experimentally and numerically $[1-6,6-9]$. There, the situation is rather simple because the grain-grain interactions, including the cohesion due to liquid bridges, are mainly two-body interactions. However, as the liquid content is increased, liquid clusters become non-negligible, which induce many-body interaction via the liquid. Methods and/or models to deal with such a situation have not been established yet and still under active research

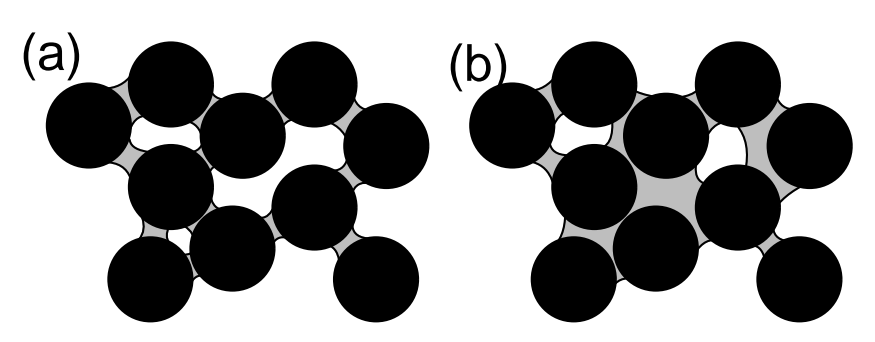

Fig. 1: Schematic description of the distribution of liquids in the wet granular materials. (a) The pendular state, where liquid bridges are formed at contact points. (b) The funicular state, where some liquid clusters that connect more than two grains are formed.

\section{$([10-15])$.}

Recent experiments show that, upon increasing the liquid content, there is a maximum in the material yield stress at rather low liquid content, in the pendular state or the funicular state [10-12]. Especially, Møller et al. [12] showed a clear increase of the shear modulus with the liquid content in the pendular state and a drop in the funicular state.

In this paper, we propose a simple phenomenological model that takes into account the many-body interaction through the liquid clusters in the funicular state. In our model, the cohesive force acts among the grains connected 
by the liquid-gas interface. As the liquid content increases, the number of grains that interact through the liquid increases, but the liquid-gas interface may decrease when the liquid cluster is formed. We show that this competition results in a maximum in the shear stress as a function of liquid-content, which may correspond qualitatively with the experimentally observed behavior of the shear modulus as a function of liquid content.

Model. - In this section, we first introduce a model for the pendular state, where the two-body cohesion due to liquid bridges is incorporated in the soft sphere model of dry grains with linear elastic force and dissipation (Discrete Element Method, DEM [16]). For the cohesive interaction, history-dependent formation of liquid bridge proposed by Schultz et al. [17] is adopted. We then extend the model to the funicular state, where some pores are filled with liquid and the many-body interactions acts among particles.

Model $P$ for the pendular state with two-body interaction. For simplicity, we consider a frictionless twodimensional system, where grains are modeled by polydisperse disks. Let us consider the interaction between particles $i$ and $j$ of diameters $\sigma_{i}$ and $\sigma_{j}$ and masses $m_{i}$ and $m_{j}$, at positions $\boldsymbol{r}_{i}$ and $\boldsymbol{r}_{j}$ with velocities $\boldsymbol{v}_{i}$ and $\boldsymbol{v}_{j}$, respectively. There is no interaction between them before they collide. Once they are in contact, i.e., $\left|\boldsymbol{r}_{i j}\right|=\left|\boldsymbol{r}_{i}-\boldsymbol{r}_{j}\right|$ becomes less than $\left(\sigma_{i}+\sigma_{j}\right) / 2$, the force on the particle $i$ by $j$ is given as a function of $\Delta \equiv\left(\sigma_{i}+\sigma_{j}\right) / 2-\left|\boldsymbol{r}_{i j}\right|$ by

$$
\boldsymbol{F}_{i j}=f(\Delta) \boldsymbol{n}_{i j}-\eta(\Delta) \boldsymbol{v}_{i j}
$$

with the normal unit vector $\boldsymbol{n}_{i j}=\boldsymbol{r}_{i j} /\left|\boldsymbol{r}_{i j}\right|$ and the relative velocity $\boldsymbol{v}_{i j}=\boldsymbol{v}_{i}-\boldsymbol{v}_{j}$, as long as $\left|\boldsymbol{r}_{i j}\right|<\alpha\left(\sigma_{i}+\sigma_{j}\right) / 2$; The parameter $\alpha(\geq 1)$ determines the distance between grains at which the liquid bridge breaks, and once $\left|\boldsymbol{r}_{i j}\right|$ exceeds $\alpha\left(\sigma_{i}+\sigma_{j}\right) / 2, \boldsymbol{F}_{i j}=0$ and the pair $i, j$ do not interact until they are in contact again.

The functions $f(\Delta)$ and $\eta(\Delta)$ in the interaction of Eq.(11) are given by

$$
f(\Delta)=\max \left(k \Delta,-F_{0}\right)
$$

and

$$
\eta(\Delta)= \begin{cases}\eta_{\text {grain }} & \text { for } \Delta>0 \\ \eta_{\text {liquid }} & \text { for } \Delta<0\end{cases}
$$

We can interpret that $\Delta$ characterizes the overlap (for $\Delta>0$ ) or the spacing (for $\Delta<0$ ) between the grains. The parameter $k$ characterizes the elastic constant, $\eta(\Delta)$ is the damping parameter, and a positive constant $F_{0}$ characterizes the cohesion due to a liquid bridge 1 .

\footnotetext{
1 One might presume that the cohesive force should act even when grains are in contact. This can be dealt with, for the present frictionless system, simply by reinterpreting $\Delta$ as being measured from the overlap position where the cohesive force and repulsive force due to grain elasticity balance. The dissipation term of eq.(3) should be modified accordingly, but such a minute adjustment barely affects the overall system behavior.
}

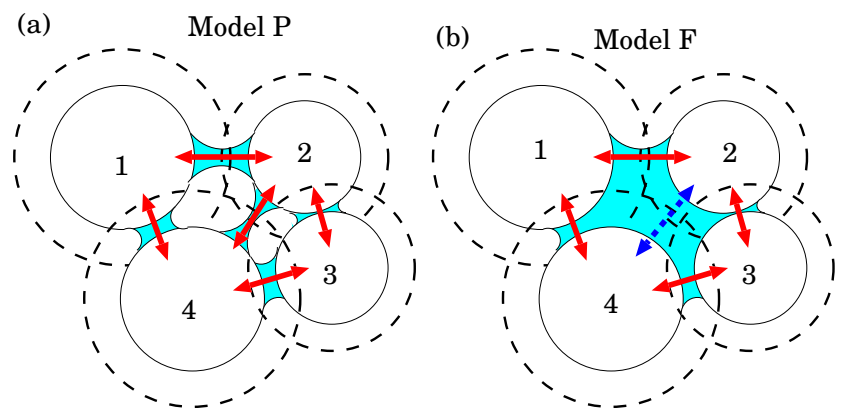

Fig. 2: Schematic description of interaction of (a) the model for the pendular state (Model P) and (b) the model for the funicular state (Model F). The solid circles show grains and the dotted line indicates the interaction range after the formation of a liquid bridge. The solid arrows indicates the pair of grains that cohesive force acts. The dotted arrow in (b) shows "inactive bonds", i.e., they are interacting via liquid cluster but the bond exert no cohesive force.

The damping parameter $\eta(\Delta)$ in eq. (3) depends on $\Delta$ because the dissipation when grains are in contact (characterized by $\eta_{\text {grain }}$ ) and the dissipation via liquid viscosity (characterized by $\eta_{\text {liquid }}$ ) has different physical origin and their values can be quite different.

Notice that the model with $\alpha=1$ corresponds to dry granular materials with neither cohesive force nor hysteresis, and $\alpha$ is greater than unity for wet cohesive grains. When $\alpha>1$, there is a hysteresis in the interaction [17], and this hysteresis causes dissipation in addition to that caused by the viscous force.

In this model for the pendular state (here we call it as Model P), all interactions are given as two-body forces. The situation is schematically illustrated in Fig. 2(a); the attractive force between the particles connected by solid arrows represents the cohesion due to liquid bridges.

Model F for the funicular state with many-body interaction. When the amount of liquid is increased enough for the system to be in the funicular state, some pores are filled by liquid, and more than two grains are connected by a single liquid-filled region. Let us consider the situation shown in Fig. 2(b), where four particles are connected by a liquid cluster. In this case, there is no liquid-gas interface that connects particles 2 and 4; in contrast to the pendular state (Fig. 2(a)), there is no direct cohesive force between them, though the cohesive force between the particles connected by solid arrows in Fig. 2(b) still tend to hold all the particles together. There should be still some effective cohesive force between the particles 2 and 4 caused by the lower pressure in the liquid phase than that in the air phase, but here we disregard this.

We model such a multi-particle effect by making the bond inactive when the bond connects the two particles between which a liquid cluster exists. The presence of the liquid cluster between two grains is determined by the simple criterion: The grains $i$ and $j$ are connected by a liquid cluster without liquid-gas interface if they are connected by 


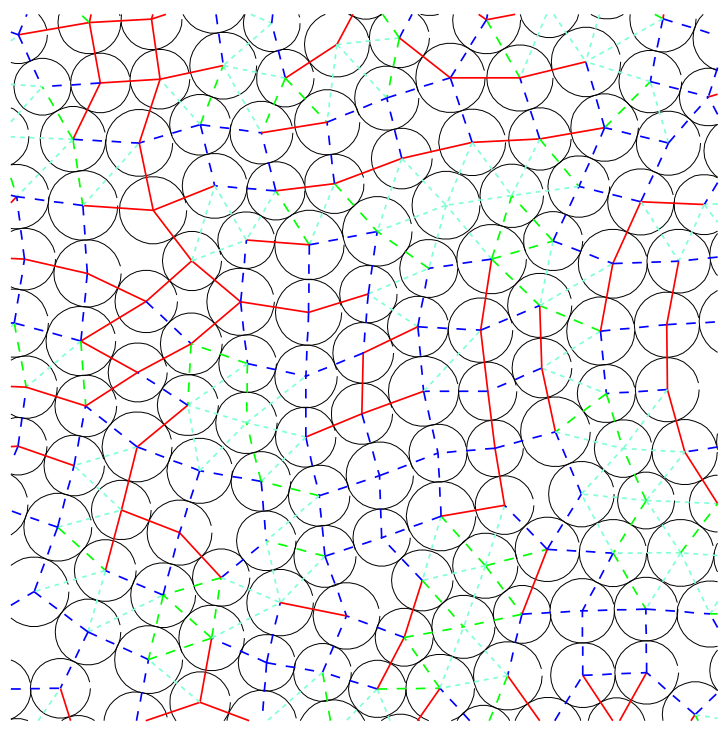

Fig. 3: Snapshot of grain-grain interactions. The interacting grains are connected by bonds. The cohesive bonds, or the bonds with $N_{i, j}<n_{t h}$ and $\Delta<0$, are shown by red solid lines, the repulsive bonds with liquid interfaces, or $N_{i, j}<n_{t h}$ and $\Delta>0$, are by blue long-dashed lines, the repulsive bonds in liquid clusters, or $N_{i, j} \geq n_{t h}$ and $\Delta>0$, are by green longdashed lines, and the inactive bonds, or $N_{i, j} \geq n_{t h}$ and $\Delta<0$, by a light blue short-dashed line.

a liquid bridge and the number of grains $N_{i, j}$ that are connected to both $i$ and $j$ by liquid bridges is larger or equal to a certain threshold value $n_{t h}$. The liquid bridge is the same as defined in the previous subsection, and we take $n_{t h}=2$ for the two-dimensional system. If two grains are connected by a big cluster without liquid-gas interface, the liquid bridge between them is inactive and no cohesive force acts through. An example in two-dimensional system with $n_{t h}=2$ is given in Fig. 2(b).

With this criterion, the force on grain $i$ by $j$ is given not by Eq. (1) but by

$$
\begin{aligned}
\boldsymbol{F}_{j i}= & f(\Delta)\left[\Theta(f(\Delta))+(1-\Theta(f(\Delta))) \Theta\left(n_{t h}-N_{i, j}\right)\right] \boldsymbol{n}_{i j} \\
& -\eta(\Delta) \boldsymbol{v}_{i j}
\end{aligned}
$$

where $f(\Delta)$ is given by Eq. (2) and $\Theta(x)$ is the Heaviside step function defined as $\Theta(x)=1$ for $x>0$ and $\Theta(x)=0$ for $x \leq 0$. We call this model for the funicular state as Model F here. Note that it is straightforward to extend this model to a three-dimensional system by choosing $n_{t h}=3$.

Figure 3 illustrates an example of the interaction network in Model F. The interacting grains are connected by bonds. The cohesive bonds, or the bonds with $N_{i, j}<n_{t h}$ and $\Delta<0$, are shown by red solid lines, the repulsive bonds with liquid interfaces, or $N_{i, j}<n_{t h}$ and $\Delta>0$, are by blue long-dashed lines, the repulsive bonds in liquid clusters, or $N_{i, j} \geq n_{t h}$ and $\Delta>0$, are by green long-dashed lines, and the inactive bonds, or $N_{i, j} \geq n_{t h}$ and $\Delta<0$, by a light blue short-dashed line. We can

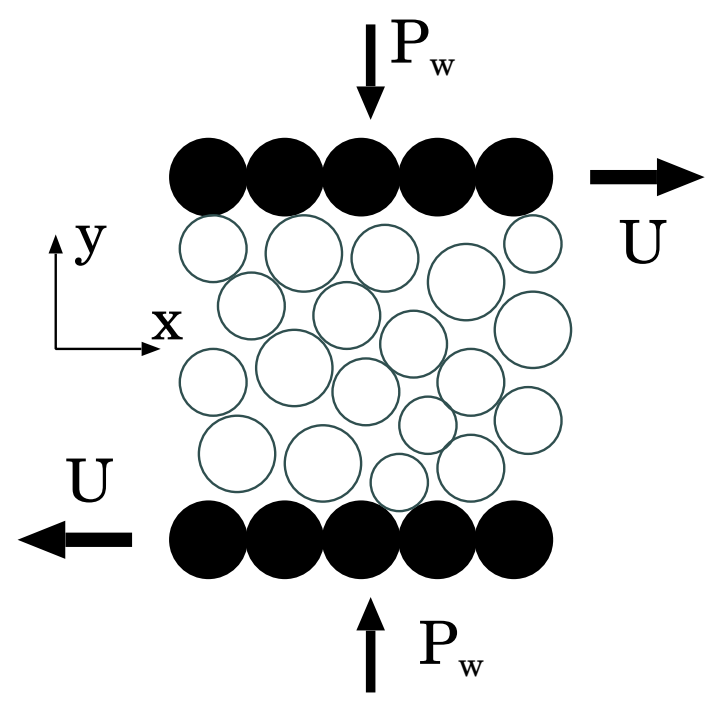

Fig. 4: Schematic description of the simulated configuration.

see several clusters of particles connected by bonds with $N_{i, j} \geq n_{t h}$, which corresponds to liquid clusters.

In this model, the parameter $\alpha$ for the interaction range represents the liquid content. In Model $\mathrm{P}$, increasing $\alpha$ just increases the number of cohesive bonds. In Model $\mathrm{F}$, on the other hand, not only the number of interacting particles increases, but also the fraction of bonds that are surrounded by other bonds $\left(N_{i, j} \geq n_{t h}\right)$ increases; such bonds exert no cohesion. Thus, the larger $\alpha$ corresponds to the case with more liquid content.

We now examine how the competition between these effects of increasing $\alpha$ in Model $\mathrm{F}$ affects the response of the wet granular assembly against shear.

Simulation Setup. - We mainly focus on Model F under shear, but we also present the results of Model P for comparison.

The grains are modeled by polydisperse disks whose diameters are uniformly distributed between $0.8 \sigma$ to $\sigma$, and all the grains have the same mass $m$. The system is periodic in the $x$ direction. In the $y$ direction, there are two parallel rough walls of mass $M$, each of which consists of $N_{w}$ particles with diameter $\sigma$ glued without spacing (Fig. 4. the total length of the wall $L=\sigma N_{w}$ ).

The top (bottom) wall is moving in the $x$ direction at a velocity $U(-U)$ in order to realize a shear flow with velocity gradient in the $y$ direction (Fig. 44). A constant pressure $P_{w}$ is applied to the walls in the $y$ direction, and the $y$ coordinate of the top (bottom) wall $Y_{t}\left(Y_{b}\right)$ obeys the equation of motion $M \ddot{Y}_{t}=F_{w, t}(t)-P_{w} L\left(M \ddot{Y}_{b}=\right.$ $\left.F_{w, b}(t)+P_{w} L\right)$, where $F_{w, t}(t)\left(F_{w, b}(t)\right)$ is the $y$-component of the force exerted from particles on the upper (bottom) wall at time $t$.

For Model F, we also need to decide whether there is liquid bridges among grains that consist the wall; That affects the formation of the liquid cluster near the wall. 
We initially impose that a neighboring pair of grains on the wall has a liquid bridge with the probability $1 / 2$, and the bridges among wall grains do not disappear or newly created during the simulation.

Initial configurations for simulations are prepared as follows; $N$ particles are distributed randomly with large enough spacing, and the wall pressure $P_{w}$ is applied without shear $(U=0)$. After waiting long enough so that all the grains are at rest, we start to shear the assembly at a constant $U$.

The data are collected in the steady states, where both the average kinetic energy of grains and the the $y$ coordinates of the walls become almost constant.

The parameters are given in the dimensionless form with the unit length $\sigma$, unit mass $m$, and the unit time scale $\sqrt{100 m \sigma^{2} / F_{0}}$ in the following. In this unit, we choose the elastic constant $k$ to be $5 \times 10^{4}$, the viscous coefficient due to grain contact $\eta_{\text {grain }}$ to be 10 (which gives the restitution coefficient $e_{n}$ for dry grains $(\alpha=1)$ around 0.9$)$, and the viscous coefficient due to liquid bridge $\eta_{\text {liquid }}$ to be 1 . In this paper, we focus on the situation where the relative velocity of particles is $O(1)$, thus the viscous force is about $1 / 100$ of the cohesive force. Also, note that the particle deformation is typically less than $1 \%$ of its diameter under the force applied in the simulations.

We use the wall mass $M=N_{w} m$, and several values of the wall pressure $P_{w}$. The system size are mostly $L=40$ and the number of particles in the system $N=1600 \mathrm{un}$ less otherwise noted. In most of the cases, the wall velocity $U$ is chosen to give the shear rate $\dot{\gamma} \approx 1$ in the middle of the system, and smaller/larger $U$ are also examined to see the tendency. The liquid-content dependence is studied by changing the interaction range $\alpha$. The secondorder Adams-Bashforth method and the trapezoidal rule are used to integrate the equations for the velocity and position, respectively, with a time step for integration $d t=10^{-4}$.

Simulation Results. - We will confirm that the system can achieve plain shear flows first, and then we will investigate the effect of liquid content on the model behavior by changing the interaction range $\alpha$.

First we check whether a plain shear flow can be realized in the present simulation. Figure 5 (a) and (b) shows the velocity profile and the packing fraction for Model $\mathrm{F}$ with $U=18, P_{w}=100$, and $\alpha=1.3$. From Fig. [5(a), we see that the realized shear rate is almost constant with $\dot{\gamma} \approx 1$, whereas some spatial structure can be seen in the packing fraction in Fig. 5(b). Similar profiles has been obtained with various $\alpha$ and $P_{w} \geq 50$ for Model F. However, we found that, when the wall pressure $P_{w}$ is too weak and/or the system size is too big, most of the grain may stick to one of the walls because of the cohesion, and the velocity gradient is confined close to the other wall. This tendency is stronger for Model $\mathrm{P}$ with large $\alpha$ and large enough system (Fig 5 (c) solid line $(\mathrm{L}=40))$. When the system is small enough (Fig. 5(c) dashed line $(\mathrm{L}=20)$ ), the plain
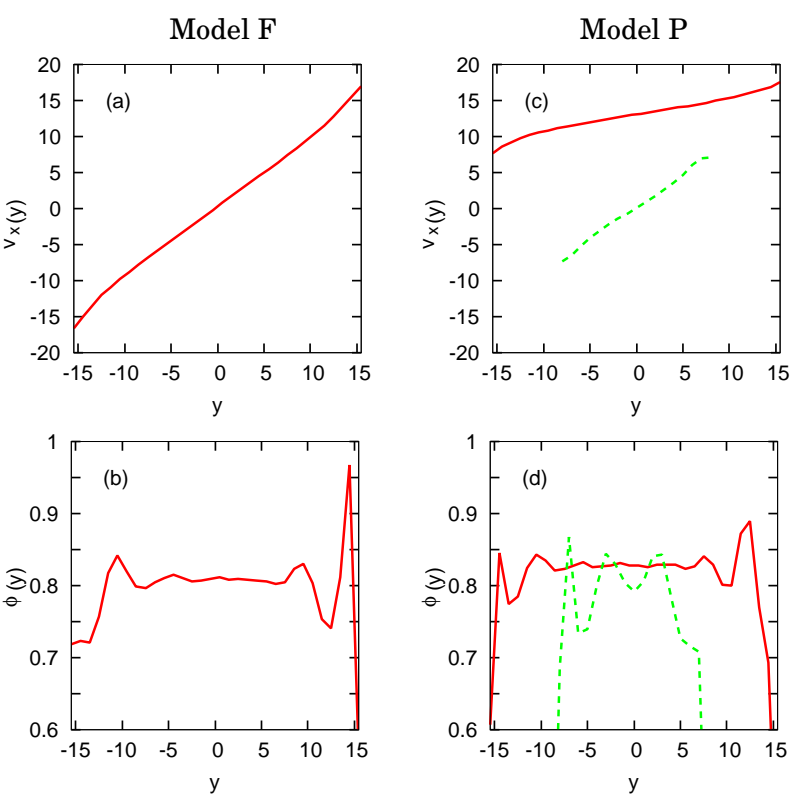

Fig. 5: (a) and (b): The velocity (a) and the packing fraction (b) profile of the system with $U=18$ for Model F with $\alpha=1.3$ and $P_{w}=100$. In (a), we can see that the shear rate $\dot{\gamma}$ is almost constant over the system with $\dot{\gamma} \approx 1$, while a spatial structure can be seen in the packing fraction profile near the boundary. (c) and (d): The velocity (c) and the packing fraction (d) profile of the system for Model $\mathrm{P}$ with $\alpha=1.3$ and $P_{w}=100$. The data for $L=40$ and $U=18$ is shown by solid lines, and the data for $L=20$ and $U=9$ is shown by dashed lines.

shear can be obtained for Model $\mathrm{P}$ also. In the following we focus on the parameter range where plain shear flow can be realized approximately.

The interaction range $\alpha$ dependence of the shear stress $S$ is shown in Figure 6 in the case of $P_{w}=100$ for Model F and Model $\mathrm{P}$ with $\dot{\gamma} \approx 1$. (for Model $\mathrm{F}, U=18$ is used, while for Model $\mathrm{P}$ the small system with $L=20$ and $N=400$ with $U=9$ is studied to avoid the nonuniform shear). Note that, for $\alpha=1$ (no cohesion due to liquid bridge), both models should give the same results. In Model $\mathrm{P}$, the shear stress increases with $\alpha$, because larger $\alpha$ simply results in more cohesive interactions. In Model F, on the other hand, $S$ shows a maximum around $\alpha=1.2$.

The maximum of the shear stress $S$ as a function of $\alpha$ in Model F can also be seen for various values of pressure $P_{w}$, but the location of the maximum changes as can be seen in Fig. 7(a). The packing fraction $\phi$ at the middle of the system, is plotted against $\alpha$ for various $P_{w}$ in Fig. 7(b); The packing fraction is higher for larger $P_{w}$ as expected, but the dependence on $\alpha$ is rather weak.

The existence of the maximum in the shear stress should be due to the competition of the increasing interacting particles and the liquid-cluster formation which reduces the cohesive interaction. To see this more directly, the average number of interacting particles ("bonds") per particle $N_{\text {int }}$ and the number of cohesive bonds (i.e., the bond 


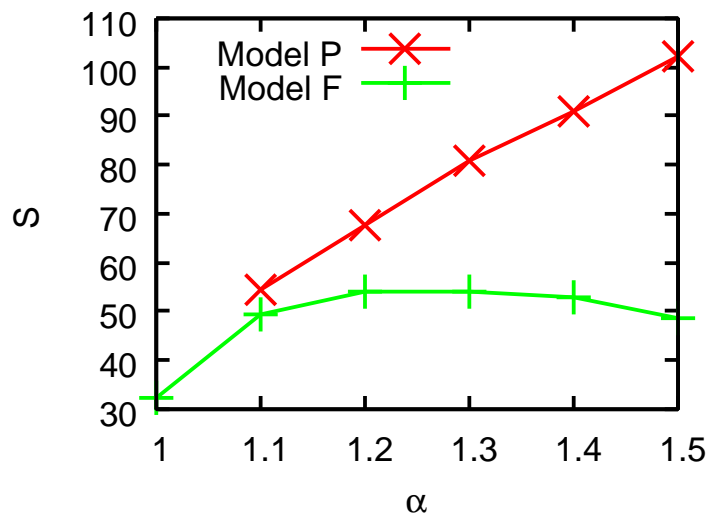

Fig. 6: The interaction range $\alpha$ dependence of the shear stress $S$ in the case of $P_{w}=100$ for Model $\mathrm{F}$ and Model $\mathrm{P}$ with $\dot{\gamma} \approx 1$. (for Model F, $U=18$ is used, while for Model $\mathrm{P}$ the small system with $L=20$ and $N=400$ with $U=9$ is studied to avoid the non-uniform shear). For $\alpha=1$, both models give the same results.

that satisfies $N_{i, j}<n_{t h}$ and $\Delta<0$ ) per particle $N_{c}$ are shown in Fig. 7(c) and (d), respectively. We can see that, even though the interacting particles increase with $\alpha$, the cohesive bonds has a maximum as a function of $\alpha$. The location of the maxima are found to be slightly different between the shear stress $S$ and the number of cohesive interactions $N_{c}$, but they have a similar trend that the maximum moves towards smaller $\alpha$ for larger $P_{w}$. We also found that $N_{c}$ is actually larger for smaller $P_{w}$ although $N_{\text {int }}$ is larger for larger $P_{w}$. This can be understood as an effect of the packing fraction $\phi$, i.e., for larger $P_{w}$, the packing fraction $\phi$ increases (Fig. 7b), which results in larger $N_{\text {int }}$ but $N_{c}$ decreases because many of the cohesive interactions become inactive as $N_{i, j}$ tends to exceed $n_{\text {th }}$ more easily.

The decrease of the cohesive bond upon increasing $\alpha$ should be a direct result of the increasing size of liquid clusters. We characterize the liquid cluster size by $N_{\text {clus }}$, the number of particles connected by bonds with $N_{i, j} \geq n_{t h}$. The cluster size distribution $P\left(N_{\text {clus }}\right)$ is shown in Fig. 8 for $P_{w}=100$ and $\dot{\gamma} \approx 1$. We find that the distribution show exponential decay for small $\alpha$, but the decay becomes slower as $\alpha$ increase, and shows power-low like behavior at around $\alpha \approx 1.4$, beyond which we get a finite probability to find a cluster as big as the system size. The cluster size distribution depends on the pressure $P_{w}$ and the shear rate $\dot{\gamma}$, but the tendency to have an exponential decay for small $\alpha$ and the slower decay for larger $\alpha$ is seen for all the cases (data not shown).

Summary and Discussion. - We proposed a simple model for the wet granular media in the funicular state. We assumed that the cohesive force acts between the particles connected by the liquid-gas interfaces but not those in a liquid cluster. We examined the model behavior upon changing the liquid content, which is represented by the
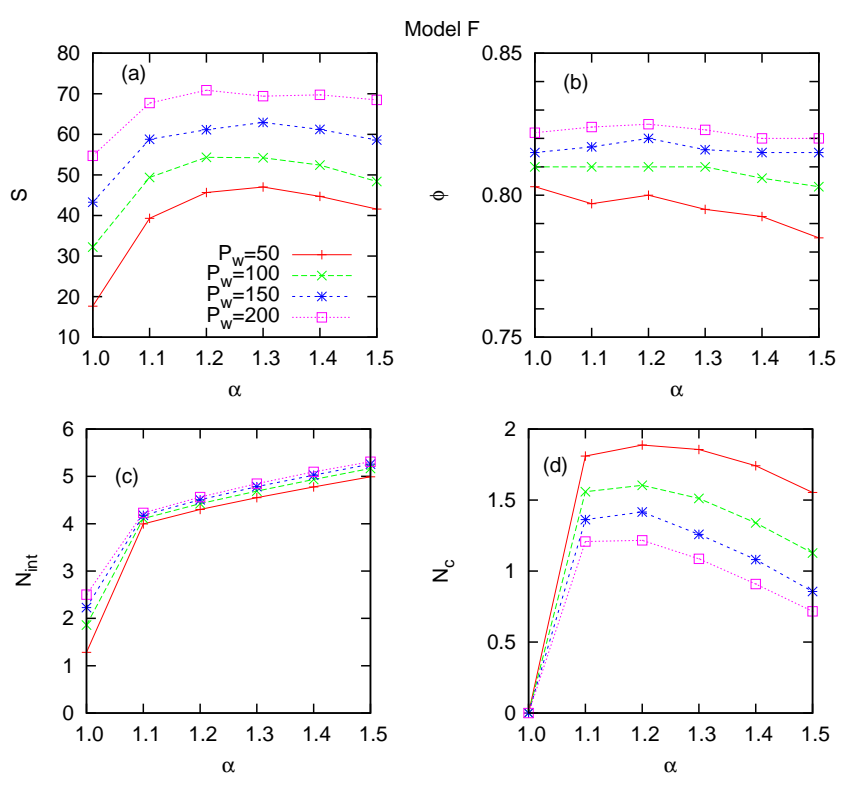

Fig. 7: The interaction range $\alpha$ dependence of the shear stress $S$ (a), the packing fraction $\phi(\mathrm{b})$, the number of bonds per particle $N_{\text {int }}$ (c), and the number of cohesive bonds per particle $N_{c}$ (d) in Model F for various pressure $P_{w} . U=18$, which gives $\dot{\gamma} \approx 1$.

interaction range parameter $\alpha$. The shear stress $S$ in a steady shear flow was demonstrated to show a maximum at a certain value of $\alpha$. The existence of the shear stress maximum is a result of competition between the two effects upon increasing the liquid content, i.e., the increase of interacting bonds, which increases the shear stress, and the formation of liquid clusters, which reduces the cohesive interaction, thus reduces the shear stress.

In experiments, the shear modulus has been demonstrated to show a maximum upon increasing the liquid content [12]. The measurements have been done for small enough stain at finite frequencies using rheometer. The observed shear modulus is fairly independent of the frequency in the range between $0.01 \mathrm{~Hz}$ and $10 \mathrm{~Hz}$, and found to show maximum values around the transition regime between the pendular and the funicular regimes for various granular materials. This experimentally observed maximum in the shear modulus may be interpreted as a result of the competition between the formation of liquid bonds and liquid clusters discussed above.

Another example of experimental observation is on the rotating drum [9]. Within the pendular state, it has been shown that the surface angle increases as the liquid volume increases at the low rotation rate, while at the high rotation rate the angle decreases with the liquid volume. The behavior at the low rotation rate is consistent with that of our Model P, but that at the high rotation rate is not. The latter case seems to indicate that the lubrication and viscous forces are important at higher rotation rate, and the rheological properties may not be simulated by simple two-body cohesions. 


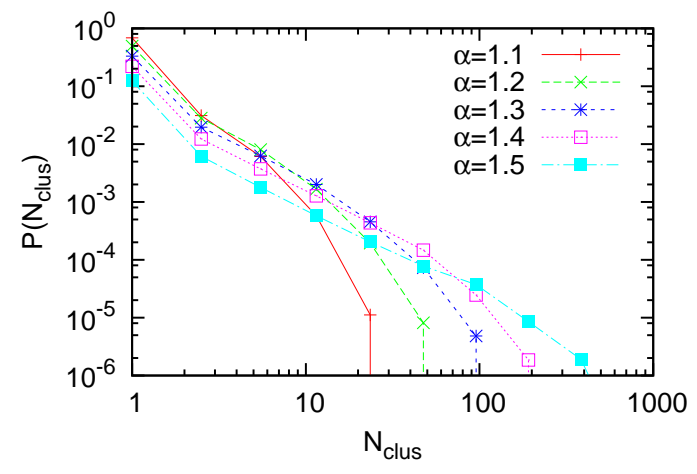

Fig. 8: The cluster size distribution $P\left(N_{\text {clus }}\right)$ with $P_{w}=100$ and $\dot{\gamma} \approx 1$ for various interaction range $\alpha$.

Before concluding, let us discuss limitations and possible extensions of our model.

Firstly, the lubrication effect is not included in the present model, and that will be important for high shear rate cases as mentioned above.

Secondly, the present model should not be valid for both limiting cases of the small liquid content and the fully saturated regime. In the former case, the friction should become important for both the sliding [5] and the rolling [18-20] motions because the cohesive force pulls the particles together to contact even without external pressure while the liquid lubrication suppresses the friction effect for larger liquid content. In the fully saturated regime, the rheology will be determined by the hydrodynamic interaction, which is not included in the present model.

Lastly, the liquid content is only represented by the parameter $\alpha$, and its conservation is not taken into account even though this may affect the configuration of the interaction network. The model for the pendular state proposed by Richefeu et al. $[7,8]$ is one of the possible approach to include the liquid conservation effectively, where a liquid volume dependent interaction has been adopted and the liquid is redistributed to each liquid bridge according to a simple rule along the time evolution. This model showed the saturation of cohesion effects as a function of the liquid content. Thus, if one add the liquid cluster criterion to it as in our model, the drop of the cohesion effect at high liquid content will be also observed because the liquid cluster will reduce the cohesive bonds. It will be interesting to see how the behavior of the model changes by including these effects.

Acknowledgment. - This work is partially supported by the grant-in-aid (21540418) by Japan Society for the Promotion of Science (JSPS).

\section{REFERENCES}

[1] Mitarai N. and Nori F., Adv. Phys. , 55 (2006) 1.

[2] Herminghaus S., Advances in Physics , 54 (2005) 221.
[3] Iveson S., Litster J., Hapgood K. and Ennis B. J., Powder Technol. , 117 (2001) 3.

[4] Brewster R., Grest G., Landry J. and Levine A., Phys. Rev. E, 72 (2005) 061301.

[5] Rognon P. G., Roux J.-N., Wolf D., NaAïm M. and Chevoir F., Europhys. Lett. , 74 (2006) 644.

[6] Rognon P. G., Roux J.-N., NAAїm M. and Chevoir F., J. Fluid Mech, 596 (2008) 21.

[7] Richefeu V., Youssoufi M. E. and Radjai F., Phys. Rev. E, 73 (2006) 051304.

[8] Richefeu V., Radjai F. and Youssoufi M. E., Eur. Phys. J. E , 21 (2006) 359.

[9] Xu Q., Orpe A. V. and Kudrolli A., Phys. Rev. E, 76 (2007) 031302.

[10] Fournier Z., Geromichalos D., Heminghaus S., Kohonen M., Mugele F., Scheel M., M.Schulz, Schulz B., Schier C., Seemann R. and Skudelny A., J. Phys.: Condens. Matter, 17 (2005) S477.

[11] Lu N., Wu B. and TAN C., J. Geotech. Geoenv. Eng. , 133 (2007) 144.

[12] Møller P. C. F. and Bonn D., Europhys. Lett. , 80 (2007) 38002.

[13] Grof A., Lawrence C. and Stepanek F., J. Colloid and Interface Sci. , 319 (2008) 182.

[14] Grof A., Lawrence C. and Stepanek F., Gran. Matt. , 10 (2008) 93.

[15] Scheel M., Seemann R., Brinkmann M., Michiel M. D., Sheppard A., Breidenbach B. and HermingHAUS S., Nature Mat. , 7 (2008) 189.

[16] Cundall P. A. and Strack O. D. L., Geotechnique, 29 (1979) 47.

[17] Schulz M., Schulz B. and Herminghaus S., Phys. Rev. $E, 67$ (2003) 052301.

[18] Bartels G., Unger T., Kadau D., Wolf D. and Kertész J., Gran. Matt. , 7 (2005) 139.

[19] Gilabert F. A., Roux J.-N. and Castellanos A., Phys. Rev. E , 75 (2007) 011303.

[20] Gilabert F. A., Roux J.-N. and Castellanos A., Phys. Rev. E, 78 (2008) 031305. 